Applied Physiology, Nutrition, and Metabolism

Canadian Science Publishing Physiologie appliquée, nutrition et métabolisme

\title{
On Task: Considerations and Future Directions for Studies of Corticospinal Excitability in Exercise Neuroscience and Related Disciplines
}

\begin{tabular}{|r|l|}
\hline Journal: & Applied Physiology, Nutrition, and Metabolism \\
\hline Manuscript ID & apnm-2018-0123.R1 \\
\hline Manuscript Type: & Review \\
\hline Date Submitted by the Author: & 19-Apr-2018 \\
\hline Complete List of Authors: & Kalmar, Jayne; Wilfred Laurier University, \\
\hline $\begin{array}{r}\text { Keyword: } \\
\text { Is the invited manuscript for } \\
\text { consideration in a Special } \\
\text { Issue? : }\end{array}$ & $\begin{array}{l}\text { corticospinal excitability, task-dependent, transcranial magnetic } \\
\text { stimulation, spinal excitability, cortical excitability }\end{array}$ \\
\hline \multicolumn{2}{|c}{} \\
\hline
\end{tabular}

\section{SCHOLARONE" \\ Manuscripts}


1 Title: On Task: Considerations and Future Directions for Studies of Corticospinal Excitability

2 in Exercise Neuroscience and Related Disciplines

4 Author: Jayne M. Kalmar

5 Wilfrid Laurier University, Department of Kinesiology and Physical Education

6 Waterloo, ON, Canada

7 ikalmar@wlu.ca

8

9

10

11

12

13 


\section{ABSTRACT:}

15 Over the last few decades, transcranial magnetic stimulation (TMS) has emerged as a

16

17

18

19

20

21

22

23

24

25

26

27

28

29

30

31 Keywords: corticospinal excitability, task-dependent, transcranial magnetic stimulation, spinal 32 excitability, cortical excitability

conventional laboratory technique in human neurophysiological research. Exercise neuroscientists have used TMS to study central nervous system contributions to fatigue, training, and performance in health, injury, and disease. In such studies, corticospinal excitability is often assessed at rest or during simple isometric tasks with the implication that the results may be extrapolated to more functional and complex movement outside of the laboratory. However, the neural mechanisms that influence corticospinal excitability are both state- and task-dependent. Furthermore, there are many sites of modulation along the pathway from the motor cortex to the muscle; a fact that is somewhat obscured by the all-encompassing and poorly-defined term "corticospinal excitability." Therefore, the tasks we use to assess corticospinal excitability and the conclusions that we draw from such a global measure of the motor pathway must be taken into consideration. The overall objective of this review is to highlight the task-dependent nature of corticospinal excitability and the tools used to assess modulation at cortical and spinal sites of modulation. By weighing the advantages and constraints of conventional approaches to studying corticospinal excitability, and considering some new and novel approaches, we will continue to advance our understanding of the neural control of movement during exercise. 
Volitional movement requires input from many parts of the motor system, including cortical motor areas (prefrontal cortex, premotor cortex, supplementary motor area, primary motor cortex), subcortical structures (cerebellum, basal ganglia), and extrapyramidal tracts

37 arising from the brainstem, that is integrated with sensory input at both segmental and supraspinal levels. Ultimately, however, the voluntary command to contract muscle is executed by corticospinal motor neurons, which input to the spinal motor neurons that activate skeletal muscle. Over the last three decades, transcranial magnetic stimulation (TMS) has been used to assess the excitability of this motor pathway from motor cortex to skeletal muscle under a variety

42 of conditions. In the relatively new discipline of Exercise Neuroscience, TMS has been used to identify fatigue-associated changes in this motor pathway (for review, see Gruet et al. 2013),

44 sex- and age-related differences in this fatigability (e.g. Yoon et al. 2015), training-induced plasticity (for review, see Kidgell et al. 2017) of the central nervous system, and corticospinal plasticity following brain injuries such as stroke (e.g. Knorr et al. 2011) and concussion (e.g.

47 Lefebvre et al. 2015). These studies report changes in what is typically referred to as corticospinal excitability during a variety of simple and reproducible motor tasks.

Although the expression corticospinal excitability is being used more widely now, a clear 50 definition of this phrase is seldom presented. While this catch-all phrase may be the most 51 appropriate way to convey findings that cannot be isolated to a single mechanism along the 52 pathway from motor cortex to corticospinal motor neuron, to alpha motor neuron, to muscle fibre 53 to recording electrode, incautious use of the phrase may result in vague and easily misconstrued 54 statements of key findings. In a PubMed search of scientific publications, the term corticospinal 55 excitability first appears in 1994 (Brouwer and Ambury 1994, Werhahn et al. 1994), nearly a 
56 decade after the first paper to describe transcranial magnetic stimulation was published by

57 Barker et al., in The Lancet in 1985 (Barker et al. 1985). The temporal relationship between the

58 emergence of TMS methodology in scientific literature and use of the term corticospinal

59 excitability is probably not coincidental, and it seems likely that increased use of this phrase

60

61

62

63

64

65

66

67

68

69

70

71 72 mechanisms. arose to accommodate TMS methodology. Wehrman et al., (1994) first used the phrase in 1994, but only once in the manuscript to state that coil orientation could be used to isolate "changes in corticospinal excitability to a cortical level", implying that the term corticospinal excitability referred to the net of cortical and spinal mechanisms. As usage of the phrase increased over the next thirty years, the implied definition became more diffuse and arguably more problematic when it became synonymous with any change in the evoked potential recorded in the muscle. For the purposes of this review, the term corticospinal excitability reflects the excitability of the pathway between the corticocortical motor axons depolarized by the magnetic stimulus and spinal motor neurons that innervate the muscle of interest, and encompasses the cortical and spinal origins of changes in the evoked potential only when changes in peripheral transmission are ruled out or accounted for. This terminology (Table 1), reflects the constraints of the tools that we use to study the system rather than distinct and easily-defined neurophysiological

\section{Corticospinal excitability: What does single-pulse TMS measure?}

When discharged over the primary motor cortex (M1), a single suprathreshold TMS

75 stimulus (Figure 1, site A) depolarizes the axons of corticocortical neurons to synaptically 76 activate corticospinal motor neurons and elicit a series of descending volleys (reviewed by 77 Bestmann and Krakauer 2015). The response to this stimulus, the motor evoked potential 78 (MEP), is recorded in the contralateral target muscle using surface electromyography (Figure 1, 
79 trace a1). The latency of this TMS-evoked potential suggests that TMS preferentially excites monosynaptic corticospinal projections to the spinal motor neuron pool (Bestmann and Krakauer 2015). Given that the MEP is elicited by synaptic input to corticospinal motor neurons and recorded in the muscle, this evoked potential is the net effect of cortical, spinal, and peripheral neuromuscular transmission. At a cortical level, the response to the stimulus will be dependent upon synaptic efficacy of the corticocortical neurons, which are depolarized by the stimulus and input to corticospinal motor neurons, the intrinsic excitability of the corticospinal motor neurons that receive the corticocortical motor neuron input, and the net excitatory and inhibitory input to the corticospinal motor neuron. These factors will determine the strength of the descending volley transmitted to the spinal motor neuron pool. Assuming that corticospinal axonal transmission failure is not a consideration, the descending volley will activate a portion of the motor neuron pool that is contingent upon the efficacy of corticospinal synaptic transmission, the intrinsic excitability of the spinal motor neuron, and net effect of inhibitory and excitability segmental and propriospinal inputs, and neuromodulatory and ionotropic descending inputs. Changes at any of these cortical and spinal sites could alter the amplitude of the resultant MEP and would thus contribute to what could reasonably be referred to as corticospinal excitability, however, the recording of the electrical response in the target muscle (the MEP) is also dependent on peripheral transmission which may be altered at the neuromuscular junction, along the sarcolemma, and between the muscle and recording electrode. Thus, if the single-pulse MEP is to be used as a global measure of "corticospinal" excitability, peripheral transmission must be taken into account (Kalmar and Cafarelli 2004). This is most often achieved by recording the maximal compound muscle action potential, or $\mathrm{M}$ wave (Figure 1, trace e) responses elicited via peripheral nerve stimulation (Figure 1, site E). Changes in the MEP that 
102 occur in the absence of changes in the $M$ wave may be attributed to events upstream to the point 103 of peripheral nerve stimulation, as a consequence of either cortical or spinal modulation.

\section{Assessing cortical contributions to corticospinal excitability}

Several approaches are used to identify a cortical origin, or at least a supraspinal origin, in TMS studies of fatigue, training, injury, aging, and pathology. The first approach is to compare the MEP elicited by a single pulse of TMS to some measure of spinal excitability derived from a potential evoked by stimulation further down the motor pathway such as the cervicomedullary evoked potential (CMEP) or the $\mathrm{H}$ reflex (Figure 1, traces $\mathrm{c}$ and $\mathrm{d}$ ). For example, fatigue is attributed to a supraspinal mechanism when MEPs elicited with single-pulse TMS decline, but CMEPs do not (Yacyshyn et al. 2017). Similarly, the effect of limb position on corticospinal excitability may be attributed to supraspinal or spinal mechanisms by reporting relative changes in MEP and CMEP. For example, biceps brachii MEP/Mwave increases and CMEP/Mwave remains unchanged at $90^{\circ}$ shoulder flexion compared to $0^{\circ}$ when the muscle is at rest. However, the same change in joint angle elicits a decrease in both MEP/M wave and CMEP/Mwave when the muscle is slightly active (Collins et al. 2017). In this case, the relative changes in MEP and CMEP suggest that changes in limb position alter corticospinal excitability through a predominantly supraspinal mechanism at rest and a spinal mechanism when the muscle is active. The spinal measures that are typically compared to a single-pulse MEP are described in detail in the following section.

Paired-pulse TMS is also used to identify intracortical mechanisms of corticospinal excitability. Briefly, two pulses are applied over the primary motor cortex through the same coil (Figure 1, site B). In the original paired-pulse paradigm (Kujirai et al. 1993), the first pulse or 
124 the "conditioning stimulus" is subthreshold and does not, delivered alone, produce an MEP in the 125 target muscle. The second pulse, or the "test pulse", is suprathreshold and does produce an 126 MEP. The time between the two stimuli determines the effect of the conditioning stimulus on 127 the test stimulus (Kujirai et al. 1993). When the interstimulus interval is less than $5 \mathrm{~ms}$, the 128 peak-to-peak amplitude of the conditioned MEP (Figure 1, trace b, red) is smaller than that of the 129 unconditioned test MEP. This effect is referred to as short interval intracortical inhibition (SICI). 130 When the interstimulus interval is $10-15 \mathrm{~ms}$, the peak-to-peak amplitude of the conditioned MEP 131 (Figure 1, trace b, green) is greater than that of the unconditioned test MEP. This effect is 132 referred to as intracortical facilitation (ICF). Other paired-pulse paradigms utilize 133 suprathreshold conditioning pulses to assess other intracortical circuits, including long interval 134 intracortical inhibition (LICI) which is elicited with an interpulse interval between 50 and $135200 \mathrm{~ms}$, and interhemispheric inhibition (IHI) which is elicited by a conditioning stimulus to the 136 opposite hemisphere 6 - $50 \mathrm{~ms}$ prior to the test pulse (for review, see Reis et al. 2008).

A third approach to identifying changes in cortical excitability, is to use a single suprathreshold TMS pulse to record the cortical silent period (Chen et al. 1999) which follows an MEP during a voluntary muscle contraction (Figure 1, trace a2). This period of electrical 140 silence is due in part to spinal mechanisms, however, there is sufficient evidence from 141 pharmacological and paired-pulse TMS studies of SICI and LICI to suggest that the later part of 142 the silent period evoked with TMS does have a cortical component (Reis et al. 2008). 143 Accordingly, this cortical silent period provides insight into intracortical inhibition during 144 voluntary activation.

While several experimental approaches provide strong support of an intracortical origin for 146 paired-pulse conditioning and the cortical silent period, the specific mechanisms (proposed 
147 circuitry, suggested interneuronal populations, and role of various neurotransmitters) are much 148 more speculative than implied in many discussions of key findings in TMS research. Thus, the 149 proposed mechanisms of paired-pulse TMS and the cortical silent period should be considered as 150 a very useful conceptual framework, but one that must nonetheless be applied with caution as 151 clearly noted by those who propose them (for review see Reis et al. 2008).

\section{Assessing spinal contributions to corticospinal excitability}

There are a number of experimental approaches used to assess the spinal component of changes in corticospinal excitability. A more detailed description of the methodologies, advantages, and limitations of these conventional measures of motor neuron excitability are reviewed thoroughly by McNeil et al. (2013). The most common measures of spinal excitability 157 in studies of corticospinal excitability include the Hoffman reflex (H reflex) and cervicomedullary evoked potentials (CMEP). In each case, the objective is to determine spinal motor neuron responsiveness to a stimulus that depolarizes the axon of a neuron with monosynaptic input to the spinal motor neuron pool. In the case of the H reflex, the axons of 161 large diameter afferents are depolarized via percutaneous electrical stimulation of the mixed nerve (Figure 1, site D). In the case of the CMEP, descending corticospinal axons are the targets of transmedullary electrical or magnetic stimulation (Figure 1, site C). Both approaches are used to assess the spinal component of corticospinal excitability, although in each case there are a few underlying assumptions that should not be dismissed (reviewed by Knikou 2008, McNeil nerve using a pulse duration that is longer (typically $1 \mathrm{~ms}$ ) and of a lower intensity than that

168 required to directly activate motor axons and produce an $\mathrm{M}$ wave (Figure 1, trace e). This low169 intensity, long-duration stimulus preferentially depolarizes the larger diameter 1a afferents 
170 (Figure 1, site D) of the mixed nerve which monosynaptically excite the homologous spinal 171 motor neuron pool. The proportion of the homologous spinal motor neuron pool activated by the 172 afferent volley will depend on the overall excitability of the motor neurons (net effect of 173 excitatory and inhibitory inputs as well the intrinsic excitability of the neuron) and is reflected by 174 the peak-to-peak amplitude of the long latency $\mathrm{H}$ reflex. The peak-to-peak amplitude of the $\mathrm{H}$ 175 reflex is plotted over a range of stimulus intensities and the maximal H reflex (Hmax, normalized 176 to the maximal $\mathrm{M}$ wave) or the slope of the ascending limb of the $\mathrm{H}$ reflex recruitment curve 177 (Hslope, normalized to the slope of the $\mathrm{M}$ wave curve) are used as measures of spinal 178 excitability. The Hmax approach is advantageous in that it provides a quick measure made in 179 between contractions or during low-level contractions during the fatigue protocol. The downside 180 of this approach is that a single $\mathrm{H}$ reflex reflects the excitability of a very narrow portion of the 181 motor neuron pool that is not likely the same as that reflected by the cortically-evoked MEP to 182 which it may be compared. While the Hslope approach provides an estimate of the excitability 183 of a much bigger proportion of the motor neuron pool, it can take upwards of 3 minutes to 184 generate a carefully constructed $\mathrm{H}$ reflex recruitment curve during which time considerable 185 recovery from fatigue will take place.

$\mathrm{H}$ reflex estimates of spinal excitability are influenced by presynaptic inhibition, are not 187 entirely monosynaptic, and are difficult to elicit in proximal muscles of the lower limb and in 188 the upper extremity (Knikou 2008). Consequently, cervicomedullary-evoked potentials (CMEP) 189 (Figure 1, trace c) elicited via transmastoid magnetic or electrical stimulation (Figure 1, site C) 190 have frequently been described as the "most appropriate" means to assess the spinal component 191 of corticospinal excitability in TMS studies (Taylor and Gandevia 2004). This view is based on 192 two key assumptions. The first is that stimulation of the brainstem at the transmastoid level 
193 (above the origin of the propriospinal interneurons at C3-C4) activates primarily corticospinal 194 axons from amongst the other descending and ascending tracts. The second assumption is that 195 the CMEP represents a predominantly monosynaptic input that, unlike the $\mathrm{H}$ reflex, is not subject 196 to presynaptic inhibition (Nielsen and Petersen 1994). While there is strong evidence from 197 collision experiments and post-stimulus time histograms of single unit responses to support these 198 assumptions (at least in the upper limb), it is important to note that other forms of stimulation at 199 the same site, albeit with different stimulation parameters, have been used to assess vestibular 200 and cerebellar contributions to motor output. Furthermore, transmastoid stimulation may excite 201 axons of spinal motor neurons of the ventral roots if the stimulation intensity is high or the 202 stimulus is not delivered in the optimal location. If this occurs, the resultant CMEP no longer 203 provides an estimate of spinal motor neuron excitability. While this error can be avoided by 204 carefully monitoring latencies, these data are not always reported. These caveats and 205 considerations are reviewed in greater depth by Taylor and Gandevia (2004). Finally, like the H 206 reflex, the portion of the motor unit pool represented by the CMEP may not be the same as that 207 which contributes to the cortically-evoked MEP given that the CMEP reflects a single 208 descending volley and TMS-elicited MEP is comprised of multiple descending volleys (Day et 209 al. 1987).

Both the $\mathrm{H}$ reflex and the CMEP provide a measure of "net excitability" of the motor 211 neuron pool that is determined by the summation of excitatory and inhibitory descending and 212 afferent input as well as intrinsic motor neuron excitability. While intrinsic motor neuron 213 excitability is frequently cited as a mechanism that may contribute to the modulation of the $\mathrm{H}$ 214 reflex and CMEP, it is rarely noted that the intrinsic excitability of a spinal motor neuron at rest 215 is quite different from the excitability of a neuron near threshold and during rhythmic firing. 
216 Neuromodulatory drive is a significant contributor to motor output, setting spinal motor neuron

217 excitability according to both state and task through the generation of persistent inward currents 218 (PIC) in motor neuron dendrites (Heckman et al. 2009). PIC amplifies and prolongs the effects 219 of synaptic input to increase firing rate and prolong the output of spinal motor neurons via 220 voltage-dependent sodium and calcium conductances, but only when the neuron is near or at 221 threshold (Gorassini et al. 2002). As such, measures of corticospinal excitability such as the $H$ 222 reflex and CMEP made with the muscle at rest, do not take this important determinant of motor 223 neuron excitability into account. Even when delivered during muscle activation, brief stimuli 224 such as those used to elicit an H-reflex or CMEP may activate the faster sodium conductance, 225 but would not activate the slower and longer-lasting calcium conductances that contribute most 226 significantly to PIC (Heckman et al. 2009).

It is possible to estimate the amplitude of PIC that contributes to the intrinsic excitability of human spinal motor neurons using intramuscular electromyography to record the firing behavior 229 of motor unit pairs (Figure 1, trace f) (Gorassini et al. 2002, Vandenberk and Kalmar 2014). To 230 obtain an estimate of PIC, the firing frequency of a low-threshold "control" motor unit is used as 231 a proxy measure of synaptic input to the spinal motor neuron pool during a triangular ramp 232 contraction. As the participant traces the ascending limb of the ramp, more synaptic input is 233 required to generate the required motor output, as indicated by an increase in control motor unit 234 firing rate (Figure 1, plot g, ). Persistent inward current is then estimated from a higher235 threshold "test" motor unit recruited later in the ramp. If this test unit did not have a PIC as a 236 source of intrinsic excitability, then one would expect it to be recruited (start firing) and 237 derecruited (stop firing) at the same level of "synaptic input" (or the same control-unit firing rate, 238 since this is our proxy measure of synaptic input). In contrast, motor units with a PIC have 
239

240

241

242

243

244

245

246

247

248

249

250

251

252

253

254

255

256

257

258

greater intrinsic excitability and can continue firing with less synaptic input once they are activated. Consequently, the control-unit firing rate (our estimate of synaptic input) will be lower at test unit decruitment than at recruitment. This difference in control-unit firing rate at test unit recruitment and decruitment $(\Delta \mathrm{F})$ provides an estimate of PIC amplitude in the test motor unit. This method does rest heavily on several assumptions (e.g. shared synaptic input, saturation of PIC in the control unit prior to test unit recruitment, and a control unit firing range which is not saturated during the ramp) which must be tested in order to include the control and test motor unit pairs in analysis (Stephenson and Maluf 2011). Furthermore, ramps with slower rates of rise and longer durations will result in $\Delta \mathrm{F}$ values that are due to intrinsic properties other than PIC that contribute to nonlinear firing behaviour (Revill and Fuglevand 2011, Vandenberk and Kalmar 2014). Paired motor unit recordings do not provide quick and easy estimates of intrinsic motor neuron excitability. Nonetheless, intrinsic properties such as PIC contribute significantly to spinal motor neuron output, and paired motor unit recordings provide a means to assess task-dependent modulation of corticospinal excitability that is intrinsic to the spinal motor neuron that is not evident at rest and not fully revealed by the brief stimuli that elicit the CMEP and $\mathrm{H}$ reflex. For example, it is possible that increased monoaminergic drive during fatiguing exercise may elicit an increase in intrinsic motor neuron excitability that compensates for both other sites of corticospinal hypoexcitability and muscle contractile failure that accompany fatigue. This hypothesis has not yet been tested.

\section{Corticospinal Excitability is State-, Time-, and Task-dependent}

Changes in corticospinal excitability reflected by the MEP may be due to both cortical and spinal mechanisms. The relative extent to which these intracortical and segmental interactions modulate MEP amplitude will depend on the state (e.g rest, arousal, fatigue, attention, injured, 
262 trained) as well as the task (e.g. rest, isometric contraction, isotonic, locomotor, required

263 precision). Furthermore, corticospinal excitability is not static during a motor task. It changes

264 rapidly leading up to, during, and after movement (Chen and Hallett 1999) such that TMS

265 provides a measure of corticospinal excitability only at the time of stimulation. Therefore, the

266 task, the state, and timing of the stimulus should be considered when designing experiments,

267 interpreting the results, and applying significant findings to behaviours that extend beyond those

268 studied. Although considering task is important in any study of the neural control of movement,

269 the ensuring discussion will largely focus on the task-dependent modulation of corticospinal

270 excitability during fatigue.

Ideally, we would assess corticospinal excitability (preferably at both cortical and spinal

272 levels) during the very motor tasks that we are most interested in, and we would make these

273 measures in multiple muscles; those that we believe to be most relevant to the task, and those

274 that are not likely to contribute to the task (to achieve some measure of specificity of changes in

275 corticospinal excitability). Furthermore, given that TMS provides a measure of corticospinal

276 excitability only at the time of stimulation, we would ideally assess it across a range of time

277 points that reflect the planning or execution phases of motor output that we contend to be most

278 pertinent to the questions that we pose. Of course, it is not possible to accomplish all of this in a

279 single experiment. Instead, corticospinal excitability is typically assessed with the muscle at

280 rest, or during visually-guided contractions at a target level of muscle activation, often in a

281 muscle operating across a single joint with movement at the joint above and below restricted or

282 completely restrained. Use of these controlled and reproducible laboratory protocols is easily

283 justified, but these paradigms do nonetheless deviate from the motor tasks and behavioural states

284 outside the lab that we are generally interested in understanding. By considering the advantages 
285 and limitations of the experimental task and the timing of stimuli used to measure corticospinal 286 excitability during the task, it is possible to design experiments that more closely approximate 287 the "real-life" motor tasks we ultimately wish to understand.

Resting measures of corticospinal excitability:

The advantage of recording corticospinal excitability at rest is that it is easier to identify and control for unintended changes in muscle activation of agonists, synergists, or antagonists 291 that would confound the interpretation of the results. However, measurements made at rest 292 without a plan to contract are measurements made in a non-motor state and it has been noted, sometimes as an afterthought, that measurements of corticospinal excitability made in relaxed muscle may not reflect corticospinal excitability during voluntary contraction. A stronger stance on this issue is probably warranted. The resting motor evoked potential provides a measure of 296 transmission from brain to muscle with very different muscle afferent input (e.g. GTO, spindle), 297 and potentially very different joint and cutaneous input that would arise from force production $298 \mathrm{and} /$ or limb movement. Furthermore, a motor evoked potential elicited at rest with no plan to 299 contract provides a measure of transmission from brain to muscle with very different input to the primary motor cortex and different descending corticospinal drive, extrapyramidal ionotropic 301 and neuromodulatory input, and propriospinal inputs to the spinal motor neuron. Thus, changes 302 in corticospinal excitability observed at rest with no plan to contract are not necessarily relevant 303 during a motor task given that segmental and descending inputs may compensate for (or 304 contribute to) changes in resting excitability once a motor task is initiated. A good solution 305 would then be to assess corticospinal excitability during a contraction. 
Not only are potentials evoked during muscle contractions reflective of a motor state, but

the variability of the evoked potentials is considerably reduced as muscle activation increases (Darling et al. 2006), thus enhancing statistical power. Furthermore, motor threshold (the minimum amount of stimulator output required to elicit evoked potentials $50 \%$ of the time) is lower during voluntary contraction than at rest. This means that a lower stimulation intensity may be employed, thus making the protocol more comfortable for the participant. What, then, are the limitations of assessing corticospinal excitability during a muscle contraction? While there are no disadvantages that preclude the effective use of this approach, there are factors that should be taken into account, including the intensity and the nature of the contraction. Differences in these factors may explain divergent results in otherwise similar studies and limit the extrapolation of the findings to all but similar motor tasks.

Consider a protocol that assesses corticospinal excitability during a submaximal contraction of a muscle maintained at a target presented to the participant as EMG or force output to be traced or maintained on a computer monitor. The idea, quite reasonably, is to control muscle activation and joint angle to permit consistent comparisons within and between experiments. However, this is a visuomotor task that requires some precision; a laboratory task that is significantly different from the movements required for most daily activities of living, sport performance, and occupational tasks to which we may wish to apply our findings. We do not normally have augmented feedback during motor tasks outside the lab; and within the lab, the nature of the feedback and the precision required to complete the task will affect measures of corticospinal excitability. For example, cortical activity is suppressed to a greater extent and for a longer time with visual feedback of finger position compared to visual feedback of the force output even when the mechanical requirements of the task and pre-stimulus muscle activation are 
330 the same (Lauber et al. 2012). Furthermore, corticospinal excitability is increased when the

331 difficulty of a force-matching task is made more difficult by increasing the sensitivity of the

332 force transducer (Pearce and Kidgell 2009), and in precise target-matching contractions

333 compared to power contractions (Schieppati et al. 1996). To further complicate the matter,

334 cortical excitability and inhibition during simple finger abduction across a single isolated joint is

335 not the same as during more natural manual tasks, such as pincer and power grip tasks and object

336 exploration. For example, when paired-pulse TMS is used to assess cortical modulation during

337 these different tasks, there is less SICI during a precision grip than during simple finger

338 abduction and at rest, but no task-dependent effect on long-interval intracortical inhibition (Opie

339 et al. 2015). When intracortical inhibition is assessed using the cortical silent period,

340 intracortical inhibition is lowest during a power grip compared to a precision grip, and highest

341 during simple finger abduction (Tinazzi et al. 2003). Finally, cortical silent periods are longest

342 (the most inhibition) during a tactile exploration task, and shortest (the least inhibition) during a

343 visually-guided ramp contraction, when compared to a tonic finger-abduction contraction (Hess

344 et al. 1999). Clearly, we must consider the "task at hand" if we are to measure corticospinal

345 excitability during muscle activation. As an aside, when acoustic startle is used elicit reticular

346 system input to both cortical and spinal motor neurons, MEPs are suppressed more during power

347 grip than during precision and finger abduction (Tazoe and Perez 2017) which highlights the

348 need to consider task-specific modulation of inputs other than the corticospinal tract during

349 movement.

Assessing corticospinal excitability during a contraction held at a submaximal target poses 351 additional challenges if the contractions are fatiguing. Experimental paradigms that elicit

352 fatigue (intentionally or not) make it difficult to ensure that a cortical stimulation is applied at a 
353 consistent level of muscle activation. In this case, the activation level required to maintain any

354 given force target may be altered by muscle contractile failure and subsequent increase in motor

355 unit recruitment (Bigland-Ritchie et al. 1986) whereas rectified or root-mean-square EMG

356 targets for muscle activation may be confounded by alterations in peripheral transmission

357 (Kalmar and Cafarelli, 2004). This matters because relatively small changes in muscle activation

358 level alter spinal motor neuron excitability and shifts the balance between cortical inhibitory and

359 facilitatory circuits (Ortu et al., 2008). Furthermore, an often-overlooked aspect of target-

360 matching fatigue protocols is that the nature of task itself may change as fatigue progresses and

361 alternative strategies are used to hold a target force. At the onset of a fatigue protocol, for

362 example, the motor task is to reach and hold a submaximal target (a visuomotor task that requires

363 some precision). If the endpoint (Tlim) for the fatigue protocol is defined as the inability to

364 reach and hold a target, then the motor task changes as Tlim approaches. The participant is no

365 longer reliant on visual feedback to produce and maintain a precise motor output. The task is

366 now to generate maximal or near maximal effort. In a recent investigation of the intracortical

367 mechanisms of fatigue, we noted that although intracortical facilitation declined over the first

$36875 \%$ of an intermittent submaximal isometric fatigue protocol, it was higher at the point of

369 failure (Sharples et al. 2016) when the participant could no longer maintain the target. In this

370 case, the task shifted from tracing a target using visual feedback to meet and maintain a precise

371 level of force at the onset of the protocol, to exerting a near maximal effort in an attempt to meet

372 the target force at the end of the protocol (a task that no longer requires precision). As such, the

373 nonlinear change in intracortical facilitation at the end of the protocol may have reflected a task-

374 dependent modulation (precision vs power) of cortical excitability rather than a state-dependent

375 modulation (fatigue). Fatigue-associated changes in intracortical facilitation were not a 
376 consequence of increased muscle activation required to meet the target force in this experiment,

377 because the intracortical facilitation was assessed with the muscle at rest just prior to the

378 contraction thus avoiding the confounding effects of muscle activation.

Excitability of the primary motor cortex changes before muscle activation, during

382 review see Chen and Hallett 1999). Measuring corticospinal excitability at rest just before a

383 planned contraction may be a good compromise between measuring corticospinal excitability at rest with no plan to contract (a non-motor state) and during contraction (when changes in muscle

385 activation may confound the results). Furthermore, measurements of corticospinal excitability made prior to movement contribute to our understanding of the preparation phase of movement.

387 We have previously assessed cortical excitability and interhemispheric inhibition in a 500-ms period prior to externally-cued, but rhythmically-repeated, force-matching contractions and found that cortical excitability and interhemispheric inhibition were both higher compared to at rest with no plan to contract (Sharples and Kalmar 2012). We then used the same precontraction protocol to assess intracortical mechanisms of fatigue and found increased ICF and

392 decreased SICI prior to contractions at the point of task failure (Sharples et al. 2016). This 393 approach provides a measure of corticospinal excitability in a motor state but without the 394 confounding effects of muscle activation targets that may change over the course of an 395 experiment that elicits neuromuscular fatigue. Nonetheless, both the timing of the stimulus and

396 the nature of the planned contraction affect even pre-movement measures of corticospinal 397 excitability. When the task is a simple reaction time task that requires the participant to contract 
398 as quickly as possible after a "Go" signal, the MEP amplitude begins to increase approximately $39980 \mathrm{~ms}$ prior to movement. If the task is self-paced rather than externally-cued, the increase in 400 MEP amplitude begins approximately 100ms prior to movement (Chen et al. 1998). A release of 401 intracortical inhibition precedes this increase in corticospinal excitability (Reynolds and Ashby 402 1999), and when an externally-cued task requires the participant decide whether or not to 403 contract, intracortical inhibition may play an even more significant role. For example, the 404 amplitude of single-pulse MEPs in the agonist and antagonist muscles (Hoshiyama et al. 1997), 405 and the homologous contralateral muscle to agonist are decreased after "no-go" commands in 406 choice reaction time tasks (Leocani et al. 2000). This reduction in corticospinal excitability is 407 attributed to enhanced SICI in the agonist muscle and a decreased LICI in agonist and 408 surrounding muscles when contraction was withheld during "no-go" trials (Sohn et al. 2002). 409 While these paired-pulse studies suggest that a cortical mechanism contributes to changes in 410 corticospinal excitability leading up to movement execution (and suppression), premovement 411 increases in corticospinal excitability also have a spinal component attributed largely to 412 reductions in presynaptic inhibition (Eichenberger and Ruegg 1984).

\section{Moving from conventional paradigms to natural motor tasks:}

A great many studies of corticospinal excitability are conducted using upper limb muscles, 415 most often recording evoked potentials from biceps brachii, first dorsal interosseous, and 416 abductor pollicis brevis, and occasionally wrist flexors or extensors. However, the tasks 417 performed by the upper limb are, of course, quite different from the tasks performed by the lower 418 limb. Depending on the overall objectives and intended application of the study, the upper limb 419 may indeed be the best model for understanding the role of corticospinal excitability in motor 
420 function. On the other hand, assessing corticospinal excitability using a finger muscle, may not

421 provide much insight into the neural control of postural and locomotor tasks carried out by lower

422 limb musculature. While it is possible to assess corticospinal excitability using single-pulse

423 TMS in the lower extremities, it is more difficult due to the deep position of the leg region of the

424 motor cortex within the interhemispheric fissure compared to the lateral and superficial hand

425 region. Nonetheless, TMS is used to assess fatigue-associated changes in corticospinal 426 excitability in isometric contractions of lower limb muscles, most often in the quadriceps (e.g.

427 Kalmar and Cafarelli 2006, Temesi et al. 2014, Finn et al. 2018) and tibialis anterior (e.g. McKay

428 et al. 1995, McKay et al. 1996, Lentz and Nielsen 2002) which are more responsive to TMS than 429 medial gastrocnemius and soleus motor neurons (Brouwer and Ambury 1994). Recording 430 cortically-evoked potentials from these muscles during locomotion is even more challenging, but 431 it is possible. Such studies suggest that corticospinal excitability of ankle dorsiflexors and 432 plantarflexors is both task-dependent (visuomotor precision stepping compared to normal gait) 433 (Schubert et al. 1999) as well as phase-dependent (Knikou et al. 2013). More recently, the 434 corticospinal contributions to rhythmic "locomotor-like" activity have been studied in both lower 435 limb (Sidhu et al. 2012) and upper limb (Forman et al. 2015, Spence et al. 2016) cycling, 436 approaches which eliminate the technical challenges of eliciting cortically-evoked and 437 transmedullary-evoked potentials during treadmill walking. The development of arm cycling 438 protocols to investigate locomotor-like activity provide an excellent example of a functionally439 relevant laboratory task that circumvents the challenges of assessing corticospinal excitability in 440 lower limb muscles, but provides valuable insight into phase-dependent (Spence, Alcock et al. 441 2016), cadence-dependent (Forman et al. 2015), and workload-dependent (Spence et al. 2016) 
442 modulation of corticospinal excitability during rhythmic and automated motor behavior 443 characteristic of locomotion.

One of the arguments for using a conventional TMS paradigm, such as delivering pulses 445 during isometric target-force contractions across a single joint, is that these protocols allow for 446 consistent and reproducible experimental conditions during the delivery of the stimuli. However, 447 when we perform natural motor tasks outside of the laboratory, there is considerable variability 448 in the way we perform a task, because of our interactions with a changing environment and shifts 449 in the strategies used to perform a task with fewer constraints. Accordingly, the variability that 450 we seek to minimize in the laboratory is an important component of natural motor tasks. 451 Designing experiments which systematically investigate this trial-to-trial variability in the 452 performance of natural motor tasks will provide valuable insight into corticospinal modulation 453 during movement.

\section{Conclusion}

Over the three decades since the first TMS article appeared in the Pubmed database, a great 456 deal of effort has gone into studying the role that corticospinal excitability may play in the neural 457 control of movement in health, disease, injury, aging, training, and fatigue in the discipline of 458 exercise neuroscience. Conventional paradigms that assess cortical and spinal excitability at rest 459 and during muscle activation have contributed significantly to our understanding of the neural 460 control of muscle. However, an appreciation of the time- and task-dependent nature of 461 corticospinal excitability should inspire us to continue devising novel and functionally relevant 462 paradigms as we move forward. Just as importantly, more explicit discussion of the how our 463 experimental tasks differ from volitional movement in a natural environment and the use of more 
464 clearly defined terminology will help to ensure that our significant findings are not applied out of 465 context.

467 Conflict of Interest:

468 The author has no conflicts of interest to report.

\section{$470 \quad$ References}

471 Barker, A. T., Jalinous, R., \& Freeston, I. L. 1985. Non-invasive magnetic stimulation of human

472 motor cortex. Lancet, 1(8437): 1106-1107. PMID: 2860322

473 Bestmann, S., \& Krakauer, J. W. 2015. The uses and interpretations of the motor-evoked

474 potential for understanding behaviour. Exp. Brain Res. 233(3): 679-689. doi:10.1007/s00221-

475 014-4183-7. PMID: 25563496

476 Bigland-Ritchie, B., Cafarelli, E., \& Vollestad, N. K. 1986. Fatigue of submaximal static

477 contractions. Acta Physiol. Scand. Suppl. 556: 137-148. PMID: 3471051

478 Brouwer, B. J., \& Ambury, P.1994. Upper extremity weight-bearing effect on corticospinal

479 excitability following stroke. Arch. Phys. Med. Rehabil. 75(8): 861-866. PMID: 8053792

480 Chen, R., \& Hallett, M. 1999. The time course of changes in motor cortex excitability

481 associated with voluntary movement. Can. J. Neurol. Sci. 26(3): 163-169. PMID: 10451737

482 Chen, R., Lozano, A. M., \& Ashby, P. 1999. Mechanism of the silent period following

483 transcranial magnetic stimulation. Evidence from epidural recordings. Exp. Brain Res. 128(4):

484 539-542. PMID: 10541749 
485 Chen, R., Yaseen, Z., Cohen, L. G., \& Hallett, M. 1998. Time course of corticospinal 486 excitability in reaction time and self-paced movements. Ann.. Neurol. 44(3): 317-325. 487 doi:10.1002/ana.410440306 PMID: 9749597

488 Collins, B. W., Cadigan, E. W. J., Stefanelli, L., \& Button, D. C. 2017. Corticospinal excitability 489 of the biceps brachii is shoulder position dependent. J. Neurophysiol. 118(6): 3242-3251. 490 doi:10.1152/jn.00527.2017. PMID: 28855295

491 Darling, W. G., Wolf, S. L., \& Butler, A. J. 2006. Variability of motor potentials evoked by 492 transcranial magnetic stimulation depends on muscle activation. Exp. Brain Res. 174(2): 376493 385. doi:10.1007/s00221-006-0468-9. PMID: 16636787

494 Day, B. L., Rothwell, J. C., Thompson, P. D., Dick, J. P., Cowan, J. M., Berardelli, A., et al. 495 1987. Motor cortex stimulation in intact man. 2. Multiple descending volleys. Brain 110 ( Pt 5): 496 1191-1209. PMID: 3676698

497 Eichenberger, A., \& Ruegg, D. G. 1984. Relation between the specific H reflex facilitation 498 preceding a voluntary movement and movement parameters in man. J. Physiol. 347: 545-559. 499 PMID: 6707967

500 Finn, H. T., Rouffet, D. M., Kennedy, D. S., Green, S., \& Taylor, J. L. 2018. Motoneuron 501 excitability of the quadriceps decreases during a fatiguing submaximal isometric contraction. J. 502 Appl. Physiol. doi: 10.1152/japplphysiol.00739.2017. PMID: 29357479

503 Forman, D. A., Philpott, D. T., Button, D. C., \& Power, K. E. 2015. Cadence-dependent changes 504 in corticospinal excitability of the biceps brachii during arm cycling. J. Neurophysiol. 114(4): 505 2285-2294. doi:10.1152/jn.00418.2015. PMID: 26289462 
506 Gorassini, M., Yang, J. F., Siu, M., \& Bennett, D. J. 2002. Intrinsic Activation of Human

507 Motoneurons: Possible Contribution to Motor Unit Excitation. J. Neurophysiol. 87(4): 1850-

508 1858. PMID: 11929906

509 Gorassini, M., Yang, J. F., Siu, M., \& Bennett, D. J. 2002. Intrinsic activation of human

510 motoneurons: reduction of motor unit recruitment thresholds by repeated contractions.

511 J.Neurophysiol. 87(4): 1859-1866. PMID: 11929907

512 Gruet, M., Temesi, J., Rupp, T., Levy, P., Millet, G. Y., \& Verges, S. 2013. Stimulation of the

513 motor cortex and corticospinal tract to assess human muscle fatigue. Neuroscience 231(0): $384-$

514 399. doi: 10.1016/j.neuroscience.2012.10.058. PMID: 23131709

515 Heckman, C. J., Mottram, C., Quinlan, K., Theiss, R., \& Schuster, J. 2009. Motoneuron

516 excitability: the importance of neuromodulatory inputs. Clin.Neurophysiol. 120(12): 2040-2054.

517 DOI: 10.1016/j.clinph.2009.08.009. PMID: 19783207

518 Hess, A., Kunesch, E., Classen, J., Hoeppner, J., Stefan, K., \& Benecke, R. 1999. Task-

519 dependent modulation of inhibitory actions within the primary motor cortex. Exp. Brain Res.

520 124(3): 321-330. PMID: 9989438

521 Hoshiyama, M., Kakigi, R., Koyama, S., Takeshima, Y., Watanabe, S., \& Shimojo, M. 1997.

522 Temporal changes of pyramidal tract activities after decision of movement: a study using

523 transcranial magnetic stimulation of the motor cortex in humans. Electroencephalogr. Clin.

524 Neurophysiol. 105(4): 255-261. PMID: 9284232 
525 Kalmar, J. M. and E. Cafarelli. 2004. Central fatigue and transcranial magnetic stimulation: 526 effect of caffeine and the confound of peripheral transmission failure. J. Neurosci. Methods 527 138(1-2): 15-26. DOI: 10.1016/j.jneumeth.2004.03.006. PMID: 15325107

528 Kalmar, J. M., \& Cafarelli, E. 2006. Central excitability does not limit postfatigue voluntary 529 activation of quadriceps femoris. J. Appl. Physiol 100(6): 1757-1764. DOI:

530 10.1152/japplphysiol.01347.2005. PMID: 16424071

531 Knikou, M. 2008. The H-reflex as a probe: pathways and pitfalls. J. Neurosci. Methods 171(1):

532 1-12. doi:10.1016/j.jneumeth.2008.02.012. PMID: 28921683

533 Knikou, M., Hajela, N., \& Mummidisetty, C. K. 2013. Corticospinal excitability during walking 534 in humans with absent and partial body weight support. Clin. Neurophysiol. 124(12): 2431535 2438. doi:10.1016/j.clinph.2013.06.004. PMID: 23810634

536 Knorr, S., Ivanova, T. D., Doherty, T. J., Campbell, J. A., \& Garland, S. J. 2011. The origins of 537 neuromuscular fatigue post-stroke. Exp. Brain Res. 214(2): 303-315. doi:10.1007/s00221-011538 2826-5. PMID: 21847644

539 Kujirai, T., Caramia, M. D., Rothwell, J. C., Day, B. L., Thompson, P. D., Ferbert, A., et al.

540 1993. Corticocortical inhibition in human motor cortex. J.Physiol. 471: 501-519. PMID:

$541 \quad 8120818$

542 Lauber, B., Leukel, C., Gollhofer, A., \& Taube, W. 2012. Time to task failure and motor cortical 543 activity depend on the type of feedback in visuomotor tasks. PLoS One 7(3): e32433.

544 doi:10.1371/journal.pone.0032433. PMID: 22427836 
545 Lefebvre, G., Tremblay, S., \& Theoret, H. 2015. Probing the effects of mild traumatic brain 546 injury with transcranial magnetic stimulation of the primary motor cortex. Brain Inj. 29(9): 547 1032-1043. doi:10.3109/02699052.2015.1028447. PMID: 25996466

548 Lentz, M., \& Nielsen, J. F. 2002. Post-exercise facilitation and depression of M wave and motor 549 evoked potentials in healthy subjects. Clin. Neurophysiol. 113(7): 1092-1098. PMID: 12088705

550 Leocani, L., Cohen, L. G., Wassermann, E. M., Ikoma, K., \& Hallett, M. 2000. Human 551 corticospinal excitability evaluated with transcranial magnetic stimulation during different 552 reaction time paradigms. Brain 123 ( Pt 6): 1161-1173. PMID: 10825355

553 McKay, W. B., Stokic, D. S., Sherwood, A. M., Vrbova, G., \& Dimitrijevic, M. R. 1996. Effect 554 of fatiguing maximal voluntary contraction on excitatory and inhibitory responses elicited by 555 transcranial magnetic motor cortex stimulation. Muscle Nerve 19(8): 1017-1024. 556 doi:10.1002/mus.880190803. PMID: 8756168

557 McKay, W. B., Tuel, S. M., Sherwood, A. M., Stokic, D. S., \& Dimitrijevic, M. R. 1995. Focal 558 depression of cortical excitability induced by fatiguing muscle contraction: a transcranial 559 magnetic stimulation study. Exp. Brain Res. 105(2): 276-282. PMID: 7498380

560 McNeil, C. J., Butler, J. E., Taylor, J. L., \& Gandevia, S. C. 2013. Testing the excitability of 561 human motoneurons. Front. Hum. Neurosci. 7: 152. doi:10.3389/fnhum.2013.00152. PMID: $562 \quad 23630483$

563 Nielsen, J., \& Petersen, N. 1994. Is presynaptic inhibition distributed to corticospinal fibres in 564 man? J. Physiol. 477(Pt 1): 47-58. PMID: 8071888 
565 Opie, G. M., Ridding, M. C., \& Semmler, J. G. 2015. Task-related changes in intracortical

566 inhibition assessed with paired- and triple-pulse transcranial magnetic stimulation. J.

567 Neurophysiol. 113(5): 1470-1479. doi:10.1152/jn.00651.2014. PMID: 25475354

568 Pearce, A. J., \& Kidgell, D. J. 2009. Corticomotor excitability during precision motor tasks. J. 569 Sci. Med. Sport 12(2): 280-283. doi:10.1016/j.jsams.2007.12.005. PMID: 18358777

570 Reis, J., Swayne, O. B., Vandermeeren, Y., Camus, M., Dimyan, M. A., Harris-Love, M., et al. 571 2008. Contribution of transcranial magnetic stimulation to the understanding of cortical

572 mechanisms involved in motor control. J. Physiol. 586(2): 325-351.

573 doi:10.1113/jphysiol.2007.144824. PMID: 17974592

574 Revill, A. L., \& Fuglevand, A. J. 2011. Effects of persistent inward currents, accommodation, 575 and adaptation on motor unit behavior: a simulation study. J.Neurophysiol. 106(3): 1467-1479. 576 PMID: 21697447

577 Reynolds, C., \& Ashby, P. 1999. Inhibition in the human motor cortex is reduced just before a 578 voluntary contraction. Neurology 53(4): 730-735. PMID: 10489033

579 Schieppati, M., Trompetto, C., \& Abbruzzese, G. 1996. Selective facilitation of responses to 580 cortical stimulation of proximal and distal arm muscles by precision tasks in man. J. Physiol. 581491 ( Pt 2): 551-562. PMID: 8866878

582 Schubert, M., Curt, A., Colombo, G., Berger, W., \& Dietz, V. 1999. Voluntary control of 583 human gait: conditioning of magnetically evoked motor responses in a precision stepping task.

584 Exp. Brain Res. 126(4): 583-588. PMID: 10422722 
585 Sharples, S. A., Gould, J. A., Vandenberk, M. S., \& Kalmar, J. M. 2016. Cortical mechanisms of 586 central fatigue and sense of effort. PLoS One 11(2): e0149026.

587 doi:10.1371/journal.pone.0149026. PMID: 26859391

588 Sharples, S. A., \& Kalmar, J. M. 2012. Modulation of cortical excitability and interhemispheric 589 inhibition prior to rhythmic unimanual contractions. J. Neurosci. Methods 210(2): 178-186.

590 DOI: 10.1016/j.jneumeth.2012.07.018. PMID: 22850557

591 Sidhu, S. K., Hoffman, B. W., Cresswell, A. G., \& Carroll, T. J. 2012. Corticospinal

592 contributions to lower limb muscle activity during cycling in humans. J. Neurophysiol. 107(1):

593 306-314. doi:10.1152/jn.00212.2011. PMID: 22013236

594 Sohn, Y. H., Wiltz, K., \& Hallett, M. 2002. Effect of volitional inhibition on cortical inhibitory 595 mechanisms. J. Neurophysiol. 88(1): 333-338. doi:10.1152/jn.2002.88.1.333. PMID: 12091558

596 Spence, A. J., Alcock, L. R., Lockyer, E. J., Button, D. C., \& Power, K. E. 2016. Phase- and 597 workload-dependent changes in corticospinal excitability to the biceps and triceps brachii during 598 arm cycling. Brain Sci 6(4). doi:10.3390/brainsci6040060. PMID: 12091558

599 Stephenson, J. L., \& Maluf, K. S. 2011. Dependence of the paired motor unit analysis on motor 600 unit discharge characteristics in the human tibialis anterior muscle. J. Neurosci. Methods 198(1): 601 84-92. PMID: 21459110

602 Taylor, J. L., \& Gandevia, S. C. 2004. Noninvasive stimulation of the human corticospinal tract. 603 J. Appl. Physiol. 1985 96(4): 1496-1503. doi:10.1152/japplphysiol.01116.2003. PMID: $604 \quad 15016794$ 
605 Tazoe, T., \& Perez, M. A. 2017. Cortical and reticular contributions to human precision and 606 power grip. J Physiol. 595(8): 2715-2730. doi:10.1113/jp273679. PMID: 27891607

607 Temesi, J., Rupp, T., Martin, V., Arnal, P. J., Feasson, L., Verges, S., et al. 2014. Central fatigue 608 assessed by transcranial magnetic stimulation in ultratrail running. Med. Sci. Sports Exerc.

609 46(6): 1166-1175. doi:10.1249/mss.0000000000000207. PMID: 24195865

610 Tinazzi, M., Farina, S., Tamburin, S., Facchini, S., Fiaschi, A., Restivo, D., et al. 2003. Task611 dependent modulation of excitatory and inhibitory functions within the human primary motor 612 cortex. Exp. Brain Res. 150(2): 222-229. PMID: 12677319

613 Vandenberk, M. S., \& Kalmar, J. M. 2014. An evaluation of paired motor unit estimates of 614 persistent inward current in human motoneurons. J. Neurophysiol. 111(9): 1877-1884. DOI: 615 10.1152/jn.00469.2013. PMID: 24523524

616 Werhahn, K. J., Fong, J. K., Meyer, B. U., Priori, A., Rothwell, J. C., Day, B. L., et al. 1994. 617 The effect of magnetic coil orientation on the latency of surface EMG and single motor unit 618 responses in the first dorsal interosseous muscle. Electroencephalogr. Clin. Neurophysiol. 93(2): 619 138-146. PMID: 7512920

620 Yacyshyn, A. F., Nettleton, J., \& McNeil, C. J. 2017. The effects of sex and motoneuron pool on 621 central fatigue. Med. Sci. Sports Exerc. 50(5):1061-1069. doi: 0.1249/MSS.0000000000001536. 622 PMID: 29283935

623 Yoon, T., Doyel, R., Widule, C., \& Hunter, S. K. 2015. Sex differences with aging in the 624 fatigability of dynamic contractions. Exp. Gerontol. 70: 1-10. doi:10.1016/j.exger.2015.07.001. 625 PMID: 26159162 


\begin{tabular}{|c|c|c|}
\hline & Definition & Most Common Measures* \\
\hline Corticospinal Excitability & $\begin{array}{l}\text { Excitability of the pathway from the cortical site of } \\
\text { depolarization (axons of corticocortical motor axons) to the } \\
\text { site of recording (muscle) that may be attributed to cortical } \\
\text { and/or spinal mechanisms when neuromuscular transmission } \\
\text { is taken into account. }\end{array}$ & $\begin{array}{l}\text { MEP (when Mwave does not change) } \\
\text { MEP/Mwave }\end{array}$ \\
\hline Cortical Excitability & $\begin{array}{l}\text { The responsiveness of corticospinal motor neurons to a } \\
\text { cortical stimulus that may be attributed to the intrinsic } \\
\text { excitability of the corticospinal motor neuron and its net } \\
\text { excitatory and inhibitory inputs. }\end{array}$ & $\begin{array}{l}\text { MEP/CMEP } \\
\text { Intracortical Facilitation (ICF) } \\
\text { Intracortical Inhibition (SICI, LICI) } \\
\text { Cortical Silent Period (CSP) }\end{array}$ \\
\hline Spinal Excitability & $\begin{array}{l}\text { The responsiveness of spinal motor neurons to an afferent or } \\
\text { descending monosynaptic input that may be attributed to its } \\
\text { intrinsic excitability, and net excitatory and inhibitory } \\
\text { descending and segmental inputs. }\end{array}$ & $\begin{array}{l}\text { CMEP } \\
\text { H reflex }\end{array}$ \\
\hline
\end{tabular}




\section{Figure Captions}

633

634

635

636

637

638

639

640

641

642

643

644

645

646

647

648

649

650

651

652

653

654

655

Figure 1: Sites of stimulation and corresponding evoked potentials most commonly used to assess corticospinal excitability in exercise neuroscience studies. Sites for magnetic (blue coil) and electrical (yellow bolt) stimulation are denoted with upper case letters together with the evoked potentials (corresponding lower case letters) recorded using surface electromyography (traces a to e). The MEP (a1) is elicited by single-pulse TMS (A) via depolarization of the axons of corticocortical motor neurons that input to the corticospinal motor neuron (CS) to provide a measure of corticospinal excitability when peripheral transmission, assessed through peripheral nerve stimulation (E) to elicit an $\mathrm{M}$ wave (e), is taken into account. Changes in corticospinal excitability may be due to cortical mechanisms or spinal mechanisms. Paired-pulse TMS (B) is used to assess intracortical facilitation using a subthreshold conditioning pulse that precedes a suprathreshold test pulse by 10 to $12 \mathrm{~ms}$ to increase the amplitude of the single-pulse MEP (green trace in b). When the conditioning pulse precedes the test pulse by 1 to $3 \mathrm{~ms}$, the singlepulse MEP amplitude is suppressed providing a measure of intracortical inhibition (red trace in b). A single-pulse suprathreshold pulse (A) elicited during voluntary activation elicits a cortical silent period (a2) to provide another measure of intracortical inhibition. Spinal excitability is assessed via transmedullary stimulation (C) of the axons of corticospinal motor neurons to elicit a CMEP (c) or by stimulation of 1a afferent neurons of the mixed nerve (D) to elicit an H reflex. These measures of spinal excitability are the net effect of excitatory and inhibitory inputs to spinal motor neurons from the corticospinal tract as well as extrapyramidal inputs from the brainstem (purple dashed lines, not all are shown) and priopriospinal inputs (not shown), the many afferent inputs to segmental circuitry (blue dashed lines, not all shown), and intrinsic excitability of the spinal motor neuron $(\alpha \mathrm{MN})$. Persistent inward current is a key mechanism of 
656 intrinsic motor neurons that cannot be assessed at rest using evoked potentials, but can be 657 estimated using intramuscular paired motor unit recordings (f) as the difference between control 658 unit firing rates $(\Delta \mathrm{F})$ at test unit recruitment and derecruitment $(\mathrm{g})$. 
Page 33 of 33
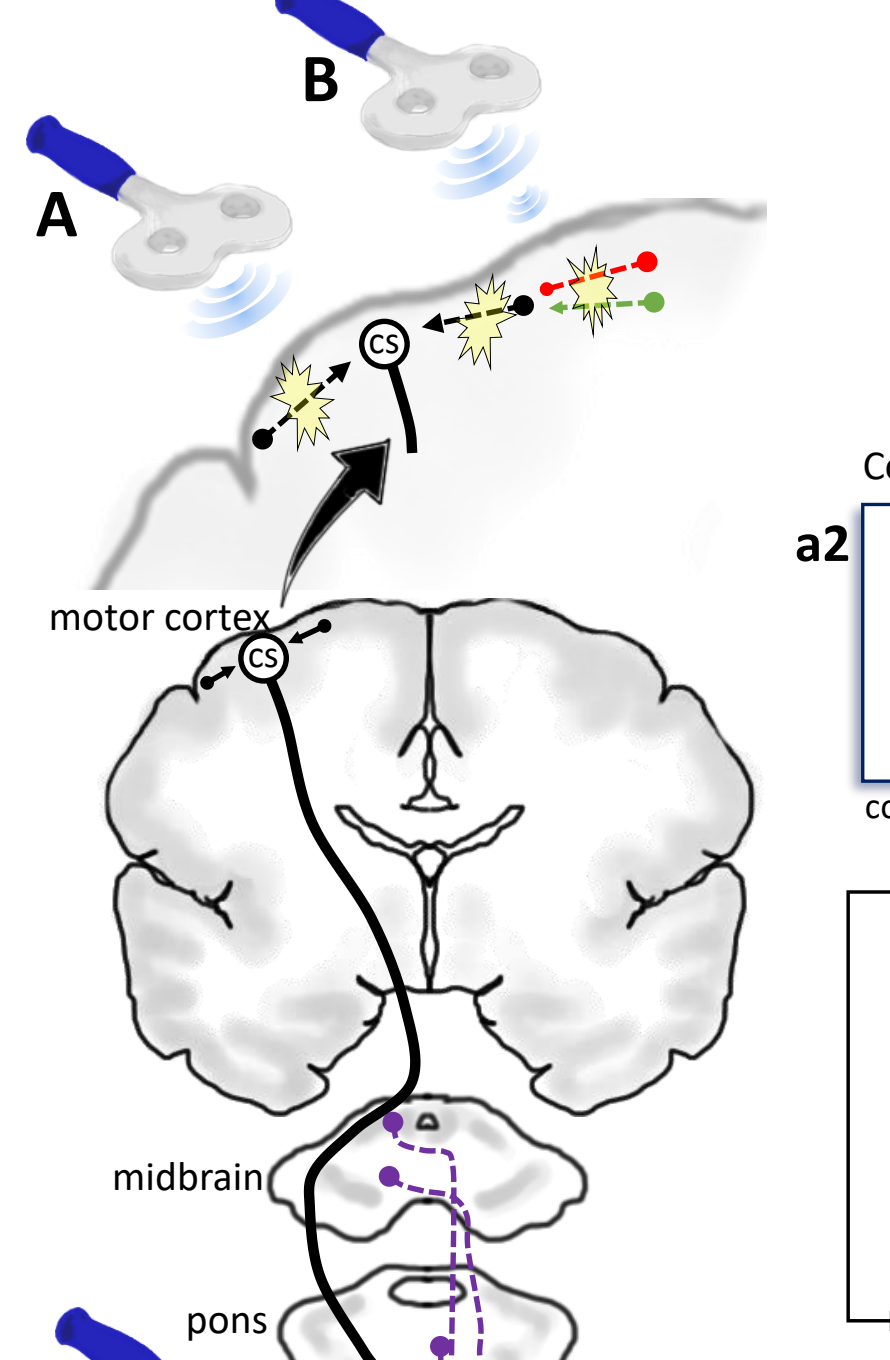